\title{
Laparoscopic removal of a foreign body migrating from the gastrointestinal tract to pancreas
}

Francesk Mulita ${ }^{1}$, Dimitris Kehagias ${ }^{1}$, Levan Tchabashvili ${ }^{1}$, Fotis Iliopoulos ${ }^{1}$, Nikolas Drakos $^{1}$, and Ioannis Kehagias ${ }^{1}$

${ }^{1}$ University General Hospital of Patras Holy Mary the Help

June 24, 2020

\begin{abstract}
A 59-year-old female presented to our hospital with epigastric pain. A computed tomography scan of the abdomen revealed a foreign body in the head of pancreas. A laparoscopic surgery was performed and the foreign body was safely removed. The foreign body was identified as a 3 -cm-long fish bone.
\end{abstract}

\section{CLINICAL VIDEO}

Laparoscopic removal of a foreign body migrating from the gastrointestinal tract to pancreas Francesk Mulita $^{1}$, Dimitris Kehagias ${ }^{1}$, Levan Tchabashvili $^{1}$, Fotis Iliopoulos $^{1}$, Nikolas Drakos $^{1}$ and Ioannis Kehagias ${ }^{1}$

${ }^{1}$ Department of General Surgery, General University Hospital of Patras, Greece

Correspondence: Francesk Mulita MD, MSc, $\mathrm{PhD}(\mathrm{c})$, Resident Surgeon at the Department of Surgery, General University Hospital of Patras, Achaia, Greece, Tel.: +30 6982785142; E-mail:oknarfmulita@hotmail.com; ORCID Id:https://orcid.org/0000-0001-7198-2628

\section{Key Clinical Message}

Laparoscopic surgery could be performed safely for the removal of a foreign body embedded in the pancreas and should therefore be preferred instead of open surgery.

\section{Case Description}

A 59-year-old female presented to the emergency department with epigastric pain. Computed tomography of the abdomen showed a linear, hyperdense, foreign body migrating from the gastrointestinal tract to pancreas. An emergency laparoscopic surgery was performed. The foreign body was safely removed laparoscopically and was identified as a 3 -cm-long fish bone (video S1). Very few cases of an ingested fish bone that migrated into the pancreas have been published in the literature so far (1). The patient recovered without complications and was discharged. Laparoscopic surgery could be performed safely for the removal of an ingested foreign body embedded in the pancreas.

Keywords: Pancreas, foreign body, laparoscopic surgery, fish bone

\section{Author contribution}


FM, DK, LT, FI and ND: contributed to the clinical data collection and prepared the case report. FM and IK: contributed to the design of the case report presentation and performed the final revision of the manuscript.

\section{References}

Mima K, Sugihara H, Kato R, et al. Laparoscopic removal of an ingested fish bone that penetrated the stomach and was embedded in the pancreas: a case report. Surg Case Rep . 2018;4(1):149. Published 2018 Dec 29. doi:10.1186/s40792-018-0559-4 